\title{
Stuttering candidate genes DRD2 but not SLC6A3 is associated with developmental dyslexia in Chinese population
}

\author{
Huan Chen ${ }^{1 \dagger}$, Guoqing Wang ${ }^{2,3+}$, Jiguang Xia ${ }^{2,3}$, Yuxi Zhou ${ }^{2,3}$, Yong Gao ${ }^{2,3}$, Junquan Xu ${ }^{2,3}$, Michael SY Huen ${ }^{4}$, \\ Wai Ting Siok ${ }^{5,6}$, Yuyang Jiang ${ }^{7}$, Li Hai $\operatorname{Tan}^{8,9^{*}}$ and Yimin Sun ${ }^{2,3,7,10^{*}}$
}

\begin{abstract}
Background: Dyslexia is a polygenic developmental disorder characterized by difficulties in reading and spelling despite normal intelligence, educational backgrounds and perception. Increasing evidences indicated that dyslexia may share similar genetic mechanisms with other speech and language disorders. We proposed that stuttering candidate genes, DRD2 and SLC6A3, might be associated with dyslexia.

Methods and results: The study was conducted in an unrelated Chinese cohort with 502 dyslexic cases and 522 healthy controls. In total, 23 Tag SNPs covering the two genes were selected for genotyping through Tagger program. Association analysis was performed on each SNP alone and in haplotypes. One SNP markers in DRD2 showed significant association with developmental dyslexia.
\end{abstract}

Conclusion: These findings indicate that polymorphism of DRD2 gene may be a risk factor of developmental dyslexia in the Chinese population.

Keywords: Dyslexia, Dopamine D2 receptor (DRD2), Solute carrier family 6, member 3 (SLC6A3), Linkage study

\section{Introduction}

Speech and language defects can be characterized as expressive (production), as receptive (comprehension) or as mixed [1]. The emergence of genetic factors implicated across multiple speech and language disorders suggested that these disorders might share similar underlying genetic mechanisms. Dyslexia, characterized by difficulties in reading and spelling despite of normal intelligence and adequate education background, is a polygenic developmental disorder affecting about $5 \%$ to $10 \%$ school-aged children in the United States $[2,3]$. Though language disorders such as dyslexia are quite different concept from speech disorders, in many cases, it is difficult to discriminate a language disorder from a speech disorder in a specific individual [1]. Goulandris

\footnotetext{
* Correspondence: tanlh@hku.hk; ymsun@capitalbio.com

${ }^{\dagger}$ Equal contributors

${ }^{8}$ Neuroimaging Laboratory, Department of Biomedical Engineering, School of Medicine, Shenzhen University, Shenzhen, China

${ }^{2}$ National Engineering Research Center for Beijing Biochip Technology,

Beijing, China

Full list of author information is available at the end of the article
}

and his colleagues conducted a comparison study between two groups of adolescents (group one with a childhood history of language impairment, group two with developmentally dyslexic) and showed that the adolescents with dyslexia were indistinguishable from those with language impairments according to their test scores of spoken and written language skills. Moreover, both dyslexic adolescents and those with language impairments showed deficiency in phonological awareness [4]. Besides, some studies revealed that motor defects may occur in both defects and therefore might explain how the two defects are connected [5,6]. As such, genes contributing to a speech disorder are recognized as putative candidate genes for a language disorder as well.

An example is the discovery of forkhead box P2 (FOXP2) and its target genes. FOXP2 gene, which encodes a neurally expressed transcription factor, was discovered through linkage analysis of a large family who had developmental verbal dyspraxia (DVD) or childhood apraxia of speech (CAS) $[7,8]$. Subsequent reports provided additional 
evidence indicating FOXP2 genetic variants as risk factors for other speech disorders and language disorders including specific language impairment (SLI) and dyslexia, and suggested that FOXP2 acts as an important hub in networks with relevance to speech and language disorders [9-14]. Contactin associated protein-like 2 (CNTNAP2) gene, a downstream target gene of FOXP2, encodes a cell-surface neurexin protein with crucial roles in brain development. Recent studies have shown that variants of the CNTNAP2 gene are risk factors for speech and language disorders including SLI, stuttering and dyslexia [15-18]. Moreover, as reviewed in a recent study, the dyslexia candidate genes including KIAA0319, doublecortin domain containing 2 (DCDC2) and roundabout homolog 1 (ROBO1) are candidate genes involved in speech sound disorder (SSD) as well [19].

Dopamine, a monoamine neurotransmitter, is released from synaptic vesicles and regulates motivation, locomotion, cognition and reward-associated functions. Dopamine transporter (DAT), the major regulator of extracellular dopamine levels in the brain, controls the amplitude, spatial and temporal dimensions of the dopaminergic responses [20]. Dysregulation of dopaminergic system has been implicated in a variety of pathological conditions such as schizophrenia, Parkinsonism, attention deficit hyperactivity disorder and drug addiction [20-22]. Dopaminergic function is considered to be critical for modulation of the neural activity of striato-thalamo-cortical circuit, which is involved in complex goal-directed or context-dependent changes in human speech and bird song output [23]. Moreover, dopaminergic system also plays an important role in maintaining linguistic functions such as speech fluency and reading, and a number of genetic polymorphisms in the system have been identified as important risk factors [24,25]. For instance, the dopamine transporter gene (SLC6A3/DAT) has been implicated in the pathogenesis of several speech and language disorders, including dyslexia and stuttering [25,26]. Besides, a dyslexia susceptibility locus (DYX7) has been identified to link to dopamine D4 receptor (DRD4) region on chromosome 11p15.5 in participants of European ancestry [27]. In the mean time, association between DRD2 and stuttering has been found in the Chinese population through highdensity genotyping [25].

Based on existing findings, dopaminergic genes DRD2 and SLC6A3 are believed to be candidate genes for dyslexia. In the present study, both genes were subjected to association and linkage analysis for dyslexia. Although the association of SLC6A3 with dyslexia has been reported in a western study [26], it would be worthwhile to validate the association of SLC6A3 with developmental dyslexia in Chinese population due to the substantial differences of linguistic and genetic backgrounds between Chinese and other western populations. Therefore, we selected tag SNPs covering above two genes and reported their association with developmental dyslexia in a large unrelated Chinese cohort.

\section{Materials and methods Subjects}

Dyslexic cases and healthy controls were selected by the two-stage procedures, as previously described [28]. This study was approved by the ethical committee of Tsinghua University School of Medicine. All study subjects were informed with written consents. First, 6,900 primary school students from Shandong province of China were invited to take a Chinese reading test, which consists of questions on character-, word-, and sentence-level. Then, we selected 1794 students with reading scores above 87 th percentile or below the 13th percentile in their grade for further investigation. Second, to assess the reading ability of these selected students, they were examined individually by a character reading test consisting of 300 Chinese characters. Then the Raven's Standard Test was applied on all students to exclude participants with intelligence deficiency. In the end, 1024 participants (502 dyslexic cases and 522 controls) were chosen for subsequent analysis.

\section{SNP markers selection and genotyping}

In total, 23 Tag SNPs covering DRD2 and SLC6A3 were selected for genotyping through Tagger program [29]. The parameters were minor allele frequency (MAF) over $5 \%$ and pairwise $\mathrm{r}^{2}$ threshold of 0.8. SNP Genotyping was performed using the Sequenom MassARRAY platform (Sequenom, San Diego, CA) in CapitalBio Corporation (Beijing, China). Briefly, saliva samples were collected and subjected to genomic DNA extraction using Oragene ${ }^{\text {ma }}$ DNA self-collection kit (DNA Genotek Inc., Ottawa, Ontario, Canada) following the manufacturer's instructions. DNA quality and quantity was determined by Nanodrop spectrophotometry (Nanodrop 1000 Spectrophotometer, Thermo Scientific, Wilmington, DE). Based on a locus-specific primer extension reaction, a locusspecific PCR reaction was designed using the MassARRAY Assay Design software package (v3.1). MALDI-TOF mass spectrometer and Mass ARRAY Type 4.0 software were applied for mass determination and data acquisition.

\section{Data analysis}

Hardy-Weinberg equilibrium (HWE) tests were performed for all SNPs individually. SNPs with a HWE P-value of less than 0.00001 (in controls) were removed. PLINK software was applied for association analysis using additive, dominant, recessive and genotype models. Linkage disequilibrium (LD) and haplotype analyses (haplotypes with above 0.01 frequence) were conducted using Haploview software (Version 4.2), as previously described [30]. The Omnibus 
ANOVA test was performed using $\mathrm{R}$ software. In Omnibus ANOVA test, the independent variable is haplotype and the dependent variable is group (dyslexia or not). In the single marker and haplotype analysis, we build the logistic regression model using genotype/haplotype as variable. We also build the logistic regression model using genotype/haplotype as variable and using age and sex as covariate. Bonferonni correction was undertaken for the 23 SNPs that were adopted into the single site association analysis.

\section{Results}

\section{Single marker analysis of SNPs within DRD2}

In DRD2 gene, we genotyped 11 Tag SNPs and found nominal association $(\mathrm{P}<0.05)$ of five SNPs with dyslexia in our cohort (Table 1). The allele $\mathrm{C}$ of rs1079727, the allele C of rs2002453, the allele C of rs2471851 and the allele $\mathrm{G}$ of rs11214607 were more frequent in patients with dyslexia than that in controls. SNP rs1079727 was significantly associated with dyslexia under recessive model $(\mathrm{P}=0.009134$, Odds Ratio, $\mathrm{OR}=1.538)$ and in homozygous genotype $(\mathrm{P}=0.008425, \mathrm{OR}=1.638)$. SNP rs2002453 was significantly associated with dyslexia in homozygous genotype $(\mathrm{P}=0.04169$, $\mathrm{OR}=1.4909)$. SNP rs17115583 showed remarkable association with dyslexia under recessive model $(\mathrm{P}=0.01465, \mathrm{OR}=0.7135)$ and in heterozygous genotype $(\mathrm{P}=0.009318, \mathrm{OR}=0.6841)$. SNP rs11214607 also revealed remarkable association with dyslexia under dominant model $(\mathrm{P}=0.01663, \mathrm{OR}=1.365)$ and in heterozygous genotype $(\mathrm{P}=0.009318$, $\mathrm{OR}=0.6841)$.

When the results were adjusted for age and sex, only SNP rs1079727 and SNP rs17115583 remained significant under the same model (rs1079727, $\mathrm{P}_{\text {adjusted }}=0.001017$, $\mathrm{OR}=1.816 ;$ SNP rs17115583, $\mathrm{P}_{\text {adjusted }}=0.01393, \quad \mathrm{OR}=$ 0.6914 ) and genotype (rs1079727, $\mathrm{P}_{\text {adjusted }}=0.001883$, $\mathrm{OR}=1.8827$; SNP rs17115583, $\mathrm{P}_{\text {adjusted }}=0.01156, \mathrm{OR}=$ 0.6686) as before adjustment. In addition, we found rs2002453 and rs2471851 achieved significant level under recessive model (rs2002453, $\mathrm{P}_{\text {adjusted }}=0.002867$, $\mathrm{OR}=1.633 ; \mathrm{SNP}$ rs2471851, $\mathrm{P}_{\text {adjusted }}=0.009401, \quad \mathrm{OR}=$ $1.67)$ and in homozygous genotype (rs2002453, $\mathrm{P}_{\text {adjusted }}=$ 0.02866, OR $=1.5311 ;$ SNP rs2471851, $P_{\text {adjusted }}=0.009509$, $\mathrm{OR}=1.7458$ ) after adjustment. Besides, rs11214607 was significantly associated with dyslexia after adjustment for age and sex in both recessive $\left(\mathrm{P}_{\text {adjusted }}=0.03885, \mathrm{OR}=\right.$ $1.53)$ and dominant ( $\left.P_{\text {adjusted }}=0.01696, \quad O R=1.398\right)$ models, the association became significant after adjustment in homozygous genotype $\left(\mathrm{P}_{\text {adjusted }}=0.008440\right.$, $\mathrm{OR}=1.8187$ ) other than in heterozygous genotype. After the Bonferonni correction for multiple comparisons, only SNP rs1079727 significantly associated with dyslexia under recessive models $\left(\mathrm{rs} 1079727, \mathrm{P}_{\text {adjusted }}=0.023, \mathrm{OR}=\right.$ 1.8160), indicating that rs1079727 is a potential SNP marker for a risk evaluation in dyslexia.

\section{Single marker analysis of SNPs within SLC6A3}

In SLC6A3, we genotyped 12 Tag SNPs and found nominal association of one SNP with dyslexia before adjustment (Table 2). The allele A of rs11133762 was more frequent in patients with dyslexia than that in controls. SNP rs11133762 was significantly associated with dyslexia under recessive model $(\mathrm{P}=0.04247, \mathrm{OR}=1.5096)$. After the adjustment of age and sex, SNP rs11133762 remained significant under recessive model $(\mathrm{P}=0.04395$, $\mathrm{OR}=1.5575)$. However, none SNPs was significantly associated with dyslexia after the Bonferonni correction. Thus, there was no significant finding for dyslexia with any of the SNP markers.

\section{Haplotype analysis}

In DRD2, haplotype analysis was conducted in three blocks (Figure 1 and Additional file 1: Table S1). Block 1 consisting of rs1079727, rs2002453, rs2471851 and rs12800853 was associated with dyslexia $(\mathrm{P}=0.022$ Omnibus test), and included one risk haplotype CCCC ( $\left.\mathrm{P}_{\text {unadjusted }}=0.00367, \mathrm{OR}=1.22\right)$. The association for haplotype CCCC remained significant after adjustment for age and sex as covariates $\left(\mathrm{P}_{\text {adjusted }}=0.0146, \mathrm{OR}=1.28\right)$. And one protective haplotype TTAC $\left(\mathrm{P}_{\text {adjusted }}=0.0327\right.$, $\mathrm{OR}=0.812$ ) was identified after adjustment for age and sex. Block 2 consisting of rs17115583 and rs11214607 was associated with dyslexia ( $\mathrm{P}=0.0387$ Omnibus test), and included one risk haplotype GG ( $\mathrm{P}_{\text {unadjusted }}=0.0425$, $\mathrm{OR}=1.21$ ). The association for haplotype $\mathrm{GG}$ also remained remarkable after adjustment for age and sex $\left(P_{\text {adjusted }}=0.0142, \mathrm{OR}=1.29\right)$. However, the above P-values failed to reach significance after the Bonferonni adjustment for multiple comparisons. Meanwhile, in SLC6A3, we identified 3 haplotypes (Figure 2), but no significant haplotype associations were found before or after adjustment (Additional file 1: Table S2).

\section{Discussion}

Dopaminergic system has a major role in fine motor movements, and dysfunction of central dopaminergic neurotransmission has been associated with the development of speech and language [25,31-33]. Here, we aimed to examine the association between dyslexia and dopaminergic genes (dopamine receptor DRD2 and dopamine transporter SLC6A3). In DRD2 gene, using a recessive model, we demonstrated that rs1079727 was significantly associated with dyslexia with the allele $\mathrm{C}$ as a risk factor after Bonferonni correction. Haplotype analysis also suggested association between the risk haplotype CCCC of rs1079727-rs2002453-rs2471851rs12800853 (Block 1) and dyslexia, but the p-value failed to reach significance after the Bonferonni correction. All four SNPs in Block1 located in the intron region of DRD2 gene, with rs1079727-rs2002453 in intron 2 and 
Table 1 Association between SNPs in DRD2 and dyslexia using the additive, dominant, genotype, and the recessive models

\begin{tabular}{|c|c|c|c|c|c|c|c|c|}
\hline & SNP & Patient & Control & $\begin{array}{c}\text { Crude } \\
\text { OR }(95 \% \mathrm{Cl})\end{array}$ & Unadjusted & $\begin{array}{l}\text { Adjusted } \\
\text { OR }(95 \% \mathrm{Cl})\end{array}$ & $\begin{array}{c}\text { Adjusted } \\
\mathrm{p} \text {-value }\end{array}$ & $\begin{array}{c}\text { Bonferroni } \\
\text { corrected p-value }\end{array}$ \\
\hline & C Allele & 930 & 975 & 1 & & 1 & & \\
\hline & T Allele & 36 & 45 & $0.8436(0.5432-1.31)$ & 0.4488 & $0.7823(0.4875-1.256)$ & 0.3091 & 1 \\
\hline & $\mathrm{CC}$ & 449 & 466 & 1 & & 1 & & \\
\hline \multirow[t]{7}{*}{ rs2440390 } & $\mathrm{CT}$ & 32 & 43 & $0.7724(0.4800-1.2427)$ & 0.2870905 & $0.7092(0.4247-1.1843)$ & 0.18903 & 1 \\
\hline & $\pi$ & 2 & 1 & $2.0757(0.1877-22.9491)$ & 0.5513879 & $1.8799(0.1540-22.9427)$ & 0.62095 & 1 \\
\hline & Dom & & & $0.802(0.5033-1.278)$ & 0.3533 & $0.7372(0.446-1.218)$ & 0.2342 & 1 \\
\hline & $\operatorname{Rec}$ & & & $2.116(0.1913-23.42)$ & 0.541 & $1.928(0.1574-23.6)$ & 0.6076 & 1 \\
\hline & T Allele & 521 & 606 & 1 & & 1 & & \\
\hline & C Allele & 445 & 414 & $1.254(1.048-1.501)$ & 0.01345 & $1.337(1.1-1.626)$ & 0.003592 & 0.0828 \\
\hline & $\pi$ & 143 & 174 & 1 & & 1 & & \\
\hline \multirow[t]{7}{*}{ rs1079727 } & $\mathrm{CT}$ & 235 & 258 & $1.10831(0.8350-1.4711)$ & 0.4765734 & $1.0949(0.8061-1.4871)$ & 0.561809 & 1 \\
\hline & $\mathrm{CC}$ & 105 & 78 & $1.6380(1.1347-2.3645)$ & 0.0084253 & $1.8827(1.2633-2.8059)$ & 0.001883 & $0.0437^{*}$ \\
\hline & Dom & & & $1.231(0.9421-1.609)$ & 0.1277 & $1.269(0.9509-1.694)$ & 0.1056 & 1 \\
\hline & $\operatorname{Rec}$ & & & $1.538(1.113-2.127)$ & 0.009134 & $1.816(1.272-2.592)$ & 0.001017 & $0.023^{*}$ \\
\hline & T Allele & 476 & 539 & 1 & & 1 & & \\
\hline & C Allele & 490 & 475 & 1.169(0.9793-1.396) & 0.0838 & $1.238(1.022-1.5)$ & 0.02946 & 0.6785 \\
\hline & $\pi$ & 123 & 136 & 1 & & 1 & & \\
\hline \multirow[t]{7}{*}{ rs2002453 } & $\mathrm{CT}$ & 230 & 267 & 0.9525(0.7049-1.2869) & 0.7511201 & 0.9340(0.6743-1.2938) & 0.681255 & 1 \\
\hline & $\mathrm{CC}$ & 130 & 104 & $1.3821(0.9692-1.9710)$ & 0.073918 & $1.5311(1.0454-2.2424)$ & 0.028655 & 0.6601 \\
\hline & Dom & & & $1.073(0.8079-1.425)$ & 0.6269 & $1.1(0.81-1.494)$ & 0.5414 & 1 \\
\hline & $\operatorname{Rec}$ & & & $1.427(1.063-1.916)$ & 0.01806 & $1.633(1.183-2.253)$ & 0.002867 & 0.0667 \\
\hline & A Allele & 565 & 641 & 1 & & 1 & & \\
\hline & C Allele & 399 & 377 & $1.205(1.004-1.446)$ & 0.04551 & $1.269(1.041-1.549)$ & 0.01864 & 0.4278 \\
\hline & AA & 165 & 198 & 1 & & 1 & & \\
\hline \multirow[t]{7}{*}{ rs2471851 } & $A C$ & 235 & 245 & $1.1510(0.8757-1.5128)$ & 0.3131888 & $1.1008(0.8188-1.4799)$ & 0.524907 & 1 \\
\hline & $\mathrm{CC}$ & 82 & 66 & $1.4909(1.0151-2.1897)$ & 0.0416912 & $1.7458(1.1457-2.6601)$ & 0.009509 & 0.2185 \\
\hline & Dom & & & $1.223(0.9439-1.585)$ & 0.1276 & $1.225(0.9262-1.62)$ & 0.1548 & 1 \\
\hline & $\operatorname{Rec}$ & & & $1.376(0.9685-1.955)$ & 0.07486 & $1.67(1.134-2.459)$ & 0.009401 & 0.2162 \\
\hline & C Allele & 921 & 959 & 1 & & 1 & & \\
\hline & T Allele & 43 & 61 & $0.7304(0.4877-1.094)$ & 0.1276 & 0.723(0.4698-1.113) & 0.1403 & 1 \\
\hline & CC & 439 & 451 & 1 & & 1 & & \\
\hline \multirow[t]{7}{*}{ rs12800853 } & $\mathrm{CT}$ & 43 & 57 & $0.7750(0.5107-1.1762)$ & 0.2310594 & 0.7807(0.4999-1.2194) & 0.276544 & 1 \\
\hline & $\pi$ & 0 & 2 & NA & & NA & & \\
\hline & Dom & & & $0.7487(0.4947-1.133)$ & 0.1711 & $0.7476(0.4802-1.164)$ & 0.1978 & 1 \\
\hline & $\operatorname{Rec}$ & & & & & & & \\
\hline & G Allele & 560 & 549 & 1 & & 1 & & \\
\hline & A Allele & 406 & 459 & $0.8636(0.7207-1.035)$ & 0.1119 & $0.8382(0.6894-1.019)$ & 0.07648 & 1 \\
\hline & GG & 167 & 138 & 1 & & 1 & & \\
\hline \multirow[t]{4}{*}{ rs17115583 } & $A G$ & 226 & 273 & $0.6841(0.5138-0.9108)$ & 0.009318 & $0.6686(0.4892-0.9138)$ & 0.01156 & 0.2668 \\
\hline & AA & 90 & 93 & $0.7997(0.5539-1.1545)$ & 0.2328118 & $0.7555(0.5102-1.1189)$ & 0.161701 & 1 \\
\hline & Dom & & & $0.7135(0.544-0.9356)$ & 0.01465 & $0.6914(0.5152-0.9279)$ & 0.01393 & 0.3197 \\
\hline & $\operatorname{Rec}$ & & & $1.012(0.7341-1.395)$ & 0.9416 & $0.9593(0.6786-1.356)$ & 0.8141 & 1 \\
\hline
\end{tabular}


Table 1 Association between SNPs in DRD2 and dyslexia using the additive, dominant, genotype, and the recessive models (Continued)

\begin{tabular}{|c|c|c|c|c|c|c|c|c|}
\hline & T Allele & 589 & 674 & 1 & & 1 & & \\
\hline & G Allele & 377 & 346 & $1.247(1.038-1.499)$ & 0.01845 & $1.324(1.084-1.616)$ & 0.005965 & 0.138 \\
\hline & $\pi$ & 177 & 225 & 1 & & 1 & & \\
\hline \multirow[t]{7}{*}{ rs11214607 } & GT & 235 & 224 & $1.3336(1.0192-1.7450)$ & 0.0358259 & $1.3111(0.9822-1.7501)$ & 0.066015 & 1 \\
\hline & GG & 71 & 61 & 1.4796(0.9969-2.1958) & 0.0517832 & $1.8187(1.1654-2.8382)$ & 0.00844 & 0.1932 \\
\hline & Dom & & & $1.365(1.058-1.761)$ & 0.01663 & $1.398(1.062-1.84)$ & 0.01696 & 0.391 \\
\hline & $\operatorname{Rec}$ & & & $1.268(0.8785-1.832)$ & 0.2045 & $1.53(1.022-2.292)$ & 0.03885 & 0.8947 \\
\hline & C Allele & 831 & 873 & 1 & & 1 & & \\
\hline & T Allele & 135 & 143 & $0.9917(0.7683-1.28)$ & 0.9488 & $0.9854(0.7477-1.299)$ & 0.9166 & 1 \\
\hline & $\mathrm{CC}$ & 358 & 373 & 1 & & 1 & & \\
\hline \multirow[t]{7}{*}{ rs12574471 } & CT & 115 & 127 & $0.9435(0.7052-1.2621)$ & 0.6950158 & 0.9483(0.6924-1.2988) & 0.740855 & 1 \\
\hline & $\pi$ & 10 & 8 & $1.3024(0.5083-3.3372)$ & 0.5821073 & $1.2050(0.4383-3.3130)$ & 0.717837 & 1 \\
\hline & Dom & & & $0.9647(0.7268-1.281)$ & 0.8037 & 0.9643(0.7098-1.31) & 0.8162 & 1 \\
\hline & $\operatorname{Rec}$ & & & $1.321(0.5171-3.376)$ & 0.5605 & $1.218(0.4417-3.359)$ & 0.703 & 1 \\
\hline & A Allele & 811 & 833 & 1 & & 1 & & \\
\hline & G Allele & 155 & 187 & $0.8478(0.6689-1.075)$ & 0.1723 & $0.8359(0.6466-1.081)$ & 0.1713 & 1 \\
\hline & $\mathrm{AA}$ & 338 & 339 & 1 & & 1 & & \\
\hline \multirow[t]{7}{*}{ rs4274224 } & $A G$ & 135 & 155 & $0.8735(0.6631-1.1507)$ & 0.3362564 & $0.8612(0.6392-1.1602)$ & 0.325729 & 1 \\
\hline & GG & 10 & 16 & $0.6268(0.2805-1.4010)$ & 0.2550209 & $0.6131(0.2577-1.4590)$ & 0.268747 & 1 \\
\hline & Dom & & & $0.8505(0.6507-1.111)$ & 0.2356 & $0.8399(0.6288-1.122)$ & 0.2374 & 1 \\
\hline & $\operatorname{Rec}$ & & & $0.6527(0.2933-1.453)$ & 0.296 & $0.6246(0.2631-1.483)$ & 0.2862 & 1 \\
\hline & C Allele & 562 & 583 & 1 & & 1 & & \\
\hline & A Allele & 402 & 437 & $0.9565(0.8039-1.138)$ & 0.6159 & $0.9669(0.8009-1.167)$ & 0.7259 & 1 \\
\hline & $\mathrm{CC}$ & 166 & 177 & 1 & & 1 & & \\
\hline \multirow[t]{7}{*}{ rs7131056 } & $A C$ & 230 & 229 & $1.0709(0.8095-1.4168)$ & 0.6313223 & $1.0434(0.7684-1.4168)$ & 0.78544 & 1 \\
\hline & $\mathrm{AA}$ & 86 & 104 & $0.8817(0.6179-1.2582)$ & 0.4877891 & $0.9131(0.6228-1.3387)$ & 0.64142 & 1 \\
\hline & Dom & & & $1.012(0.7788-1.315)$ & 0.9298 & $1.005(0.7573-1.333)$ & 0.9743 & 1 \\
\hline & $\operatorname{Rec}$ & & & $0.8478(0.6172-1.165)$ & 0.308 & $0.8876(0.6292-1.252)$ & 0.4969 & 1 \\
\hline & G Allele & 679 & 730 & 1 & & 1 & & \\
\hline & C Allele & 287 & 290 & $1.059(0.8792-1.275)$ & 0.5477 & $1.099(0.8983-1.343)$ & 0.3599 & 1 \\
\hline & GG & 245 & 273 & 1 & & 1 & & \\
\hline \multirow[t]{4}{*}{ rs72999677 } & CG & 189 & 184 & $1.14457(0.8769-1.4939)$ & 0.320458 & $1.1395(0.8519-1.5242)$ & 0.378904 & 1 \\
\hline & $\mathrm{CC}$ & 49 & 53 & $1.0302(0.6735-1.5758)$ & 0.8909049 & $1.1518(0.7287-1.8206)$ & 0.545185 & 1 \\
\hline & Dom & & & $1.119(0.8722-1.436)$ & 0.3766 & $1.143(0.8731-1.496)$ & 0.3307 & 1 \\
\hline & $\operatorname{Rec}$ & & & $0.9735(0.6461-1.467)$ & 0.8979 & $1.1(0.7044-1.716)$ & 0.6762 & 1 \\
\hline
\end{tabular}

*P $<0.05$, Bold data: adjusted $\mathrm{p}$ value $<0.05$.

rs2471851-rs12800853 in intron 1. In previous reports, SNP rs1079727 has been associated with other common childhood-onset neurodevelopmental disorders including schizophrenia (SCZ) and attention-deficit/ hyperactivity disorder (ADHD), which affected about 5\% school-aged children [34-37]. Thus the significant association between SNP rs1079727 and dyslexia as well as other neurodevelopmental disorders might be an indication of its regulatory function in gene transcription regulation. To further define the loci associated with dyslexia, we also performed an imputation analysis (data not shown), which revealed that SNP rs1076560 and rs2283265 were significantly associated with dyslexia (rs1076560, $\mathrm{P}=0.04246 ; \mathrm{rs} 2283265, \mathrm{P}=0.04196)$. The intronic SNP rs2283265 and SNP rs1076560 have been shown to affect alternative splicing of DRD2 transcript, and both SNPs were also associated with activity of the ventral striatum and prefrontal cortex during working 
Table 2 Association between SNPs in SLC6A3 and dyslexia using the additive, dominant, genotype, and the recessive models

\begin{tabular}{|c|c|c|c|c|c|c|c|c|}
\hline & SNP & Patient & Control & $\begin{array}{c}\text { Crude } \\
\text { OR }(95 \% \mathrm{Cl})\end{array}$ & $\begin{array}{c}\text { Unadjusted } \\
\text { p-value }\end{array}$ & $\begin{array}{l}\text { Adjusted } \\
\text { OR }(95 \% \mathrm{Cl})\end{array}$ & $\begin{array}{l}\text { Adjusted } \\
\mathrm{p} \text {-value }\end{array}$ & $\begin{array}{c}\text { Bonferroni } \\
\text { corrected p-value }\end{array}$ \\
\hline & C Allele & 552 & 629 & 1 & & 1 & & \\
\hline & T Allele & 386 & 363 & $1.222(1.013-1.475)$ & 0.03628 & $1.232(1.004-1.511)$ & 0.04568 & 1 \\
\hline & $\mathrm{CC}$ & 158 & 194 & 1 & & 1 & & \\
\hline \multirow[t]{7}{*}{ rs11133762 } & $C T$ & 236 & 241 & $1.2024(0.9121-1.5850)$ & 0.19106664 & $1.1500(0.8517-1.5527)$ & 0.361697 & 1 \\
\hline & $\pi$ & 75 & 61 & $1.5096(1.0141-2.2474)$ & 0.04246577 & $1.5575(1.0121-2.3967)$ & 0.043953 & 1 \\
\hline & Dom & & & $1.264(0.972-1.645)$ & 0.08041 & $1.235(0.9291-1.642)$ & 0.1461 & 1 \\
\hline & $\operatorname{Rec}$ & & & $1.357(0.9429-1.954)$ & 0.1002 & $1.46(0.9799-2.175)$ & 0.06285 & 1 \\
\hline & G Allele & 925 & 974 & 1 & & 1 & & \\
\hline & A Allele & 37 & 44 & $0.8916(0.5778-1.376)$ & 0.604 & $0.935(0.5856-1.493)$ & 0.7783 & 1 \\
\hline & GG & 445 & 468 & 1 & & 1 & & \\
\hline \multirow[t]{7}{*}{ rs3863145 } & $A G$ & 35 & 38 & $0.9687(0.6011-1.5610)$ & 0.89593367 & $1.0151(0.6043-1.7051)$ & 0.954917 & 1 \\
\hline & AA & 1 & 3 & 0.3506(0.0364-3.3758) & 0.36433775 & $0.4064(0.0394-4.1922)$ & 0.449521 & 1 \\
\hline & Dom & & & $0.9234(0.5794-1.472)$ & 0.7376 & $0.9701(0.5851-1.608)$ & 0.9063 & 1 \\
\hline & $\operatorname{Rec}$ & & & $0.3514(0.03643-3.39)$ & 0.3658 & $0.4136(0.0402-4.256)$ & 0.458 & 1 \\
\hline & C Allele & 734 & 796 & 1 & & 1 & & \\
\hline & T Allele & 232 & 218 & $1.151(0.9343-1.417)$ & 0.1867 & $1.127(0.9-1.411)$ & 0.2976 & 1 \\
\hline & $\mathrm{CC}$ & 281 & 314 & 1 & & 1 & & \\
\hline \multirow[t]{7}{*}{ rs40184 } & $\mathrm{CT}$ & 172 & 168 & $1.1440(0.8763-1.4937)$ & 0.32260995 & $1.0956(0.8214-1.4613)$ & 0.53434 & 1 \\
\hline & $\pi$ & 30 & 25 & $1.3409(0.7701-2.3350)$ & 0.29988039 & $1.3203(0.7261-2.4007)$ & 0.362334 & 1 \\
\hline & Dom & & & $1.17(0.9067-1.509)$ & 0.228 & $1.128(0.8567-1.485)$ & 0.3909 & 1 \\
\hline & $\operatorname{Rec}$ & & & $1.277(0.7396-2.204)$ & 0.3804 & $1.306(0.7249-2.355)$ & 0.3738 & 1 \\
\hline & C Allele & 939 & 987 & 1 & & 1 & & \\
\hline & T Allele & 27 & 33 & $0.8604(0.5137-1.441)$ & 0.5677 & $0.8694(0.4988-1.515)$ & 0.6214 & 1 \\
\hline & CC & 456 & 478 & 1 & & 1 & & \\
\hline \multirow[t]{7}{*}{ rs6869645 } & $C T$ & 27 & 31 & $0.9130(0.5365-1.5539)$ & 0.73717584 & $0.9431(0.5296-1.6794)$ & 0.842233 & 1 \\
\hline & $\pi$ & 0 & 1 & NA & & NA & & \\
\hline & Dom & & & $0.8845(0.5216-1.5)$ & 0.6486 & $0.9032(0.5099-1.6)$ & 0.7273 & 1 \\
\hline & $\operatorname{Rec}$ & & & NA & & NA & & \\
\hline & A Allele & 617 & 643 & 1 & & 1 & & \\
\hline & C Allele & 347 & 375 & $0.9625(0.7976-1.161)$ & 0.6899 & $0.9412(0.768-1.154)$ & 0.5595 & 1 \\
\hline & $\mathrm{AA}$ & 190 & 199 & 1 & & 1 & & \\
\hline \multirow[t]{7}{*}{ rs40358 } & $A C$ & 237 & 245 & $1.01329(0.7756-1.3235)$ & 0.92355677 & 0.9603(0.7183-1.2838) & 0.784396 & 1 \\
\hline & CC & 55 & 65 & $0.8862(0.5879-1.3360)$ & 0.56412977 & $0.8738(0.5617-1.3593)$ & 0.549582 & 1 \\
\hline & Dom & & & $0.9866(0.7644-1.273)$ & 0.9172 & $0.945(0.7171-1.245)$ & 0.6877 & 1 \\
\hline & $\operatorname{Rec}$ & & & $0.8798(0.6-1.29)$ & 0.5123 & $0.8838(0.5843-1.337)$ & 0.5587 & 1 \\
\hline & A Allele & 861 & 923 & 1 & & 1 & & \\
\hline & G Allele & 101 & 93 & $1.167(0.8654-1.575)$ & 0.3109 & $1.098(0.7937-1.52)$ & 0.5712 & 1 \\
\hline & $\mathrm{AA}$ & 385 & 418 & 1 & & 1 & & \\
\hline \multirow[t]{4}{*}{ rs10052016 } & $A G$ & 91 & 87 & $1.1356(0.8206-1.5715)$ & 0.44286861 & $1.0895(0.7663-1.5492)$ & 0.632995 & 1 \\
\hline & GG & 5 & 3 & 1.8095(0.4297-7.6209) & 0.41883352 & $1.3168(0.2851-6.0832)$ & 0.724443 & 1 \\
\hline & Dom & & & $1.158(0.8417-1.594)$ & 0.3674 & $1.098(0.7764-1.552)$ & 0.5977 & 1 \\
\hline & $\operatorname{Rec}$ & & & $1.768(0.4203-7.439)$ & 0.4369 & $1.297(0.2802-6.001)$ & 0.7396 & 1 \\
\hline
\end{tabular}


Table 2 Association between SNPs in SLC6A3 and dyslexia using the additive, dominant, genotype, and the recessive models (Continued)

\begin{tabular}{|c|c|c|c|c|c|c|c|c|}
\hline & C Allele & 645 & 677 & 1 & & 1 & & \\
\hline & A Allele & 319 & 341 & $0.9808(0.8089-1.189)$ & 0.8435 & $0.9492(0.7711-1.168)$ & 0.6232 & 1 \\
\hline & $\mathrm{CC}$ & 208 & 220 & 1 & & 1 & & \\
\hline \multirow[t]{7}{*}{ rs403636 } & $A C$ & 229 & 237 & $1.0220(0.7860-1.3288)$ & 0.87099199 & $1.0459(0.7870-1.3900)$ & 0.756883 & 1 \\
\hline & AA & 45 & 52 & $0.9153(0.5884-1.4238)$ & 0.6946183 & $0.7948(0.4921-1.2836)$ & 0.347614 & 1 \\
\hline & Dom & & & $1.003(0.7798-1.29)$ & 0.9826 & $0.9992(0.7614-1.311)$ & 0.9952 & 1 \\
\hline & $\operatorname{Rec}$ & & & $0.905(0.5946-1.377)$ & 0.6413 & $0.7838(0.4989-1.232)$ & 0.2907 & 1 \\
\hline & T Allele & 816 & 878 & 1 & & 1 & & \\
\hline & C Allele & 150 & 142 & $1.136(0.8864-1.456)$ & 0.3137 & $1.128(0.8626-1.476)$ & 0.3783 & 1 \\
\hline & $\pi$ & 344 & 379 & 1 & & 1 & & \\
\hline \multirow[t]{7}{*}{ rs2937639 } & CT & 128 & 120 & $1.1752(0.8805-1.5684)$ & 0.27303583 & $1.1803(0.8642-1.6120)$ & 0.297244 & 1 \\
\hline & CC & 11 & 11 & $1.1017(0.4717-2.5736)$ & 0.82287421 & $1.0280(0.4145-2.5496)$ & 0.952512 & 1 \\
\hline & Dom & & & $1.169(0.8837-1.546)$ & 0.2739 & $1.169(0.8636-1.582)$ & 0.3123 & 1 \\
\hline & $\operatorname{Rec}$ & & & $1.057(0.4541-2.462)$ & 0.8974 & $0.9771(0.3924-2.433)$ & 0.9603 & 1 \\
\hline & A Allele & 506 & 535 & 1 & & 1 & & \\
\hline & G Allele & 454 & 485 & $0.989(0.8238-1.187)$ & 0.9055 & $1.018(0.8355-1.24)$ & 0.8615 & 1 \\
\hline & AA & 124 & 133 & 1 & & 1 & & \\
\hline \multirow[t]{7}{*}{ rs3756450 } & $A G$ & 258 & 269 & $1.0287(0.7633-1.3864)$ & 0.85244842 & $1.0910(0.7906-1.5055)$ & 0.596073 & 1 \\
\hline & GG & 98 & 108 & $0.9733(0.6743-1.4047)$ & 0.8849171 & $1.0310(0.6914-1.5373)$ & 0.881023 & 1 \\
\hline & Dom & & & $1.013(0.7622-1.346)$ & 0.9299 & $1.073(0.7889-1.458)$ & 0.6547 & 1 \\
\hline & $\operatorname{Rec}$ & & & $0.9549(0.7023-1.298)$ & 0.7685 & $0.9685(0.6952-1.349)$ & 0.8497 & 1 \\
\hline & A Allele & 823 & 886 & 1 & & 1 & & \\
\hline & G Allele & 143 & 134 & $1.148(0.8909-1.479)$ & 0.2859 & $1.141(0.8671-1.501)$ & 0.3463 & 1 \\
\hline & $\mathrm{AA}$ & 350 & 386 & 1 & & 1 & & \\
\hline \multirow[t]{7}{*}{ rs2550946 } & $A G$ & 123 & 114 & $1.1899(0.8877-1.5951)$ & 0.24476629 & $1.2021(0.8760-1.6495)$ & 0.254198 & 1 \\
\hline & GG & 10 & 10 & $1.1029(0.4536-2.6813)$ & 0.82898896 & $1.0040(0.3872-2.6035)$ & 0.99349 & 1 \\
\hline & Dom & & & $1.183(0.8902-1.572)$ & 0.2468 & $1.186(0.8724-1.613)$ & 0.2761 & 1 \\
\hline & $\operatorname{Rec}$ & & & $1.057(0.4361-2.563)$ & 0.9022 & $0.9507(0.3652-2.475)$ & 0.9176 & 1 \\
\hline & C Allele & 595 & 615 & 1 & & 1 & & \\
\hline & A Allele & 371 & 403 & $0.9467(0.7832-1.144)$ & 0.5712 & $0.9343(0.761-1.147)$ & 0.5166 & 1 \\
\hline & $\mathrm{CC}$ & 173 & 174 & 1 & & 1 & & \\
\hline \multirow[t]{7}{*}{ rs12652860 } & $A C$ & 249 & 267 & $0.9380(0.7144-1.2314)$ & 0.64476562 & 0.9233(0.6859-1.2428) & 0.598692 & 1 \\
\hline & $\mathrm{AA}$ & 61 & 68 & $0.9022(0.6020-1.3523)$ & 0.6183072 & $0.8904(0.5778-1.3720)$ & 0.598766 & 1 \\
\hline & Dom & & & $0.9307(0.7169-1.208)$ & 0.5898 & $0.9163(0.691-1.215)$ & 0.544 & 1 \\
\hline & $\operatorname{Rec}$ & & & $0.9374(0.6472-1.358)$ & 0.7326 & $0.9203(0.6162-1.374)$ & 0.6849 & 1 \\
\hline & G Allele & 536 & 549 & 1 & & 1 & & \\
\hline & T Allele & 430 & 471 & $0.9285(0.771-1.118)$ & 0.4343 & $0.9315(0.7625-1.138)$ & 0.4869 & 1 \\
\hline & GG & 137 & 136 & 1 & & 1 & & \\
\hline \multirow[t]{4}{*}{ rs12654851 } & GT & 262 & 277 & $0.9389(0.7017-1.2564)$ & 0.67158199 & $0.9964(0.7282-1.3633)$ & 0.981816 & 1 \\
\hline & $\pi$ & 84 & 97 & $0.8597(0.5900-1.2525)$ & 0.43094129 & $0.8456(0.5610-1.2747)$ & 0.423275 & 1 \\
\hline & Dom & & & $0.9184(0.695-1.214)$ & 0.5493 & $0.9584(0.7095-1.295)$ & 0.782 & 1 \\
\hline & $\operatorname{Rec}$ & & & 0.8964(0.649-1.238) & 0.5066 & $0.8546(0.6038-1.21)$ & 0.3754 & 1 \\
\hline
\end{tabular}




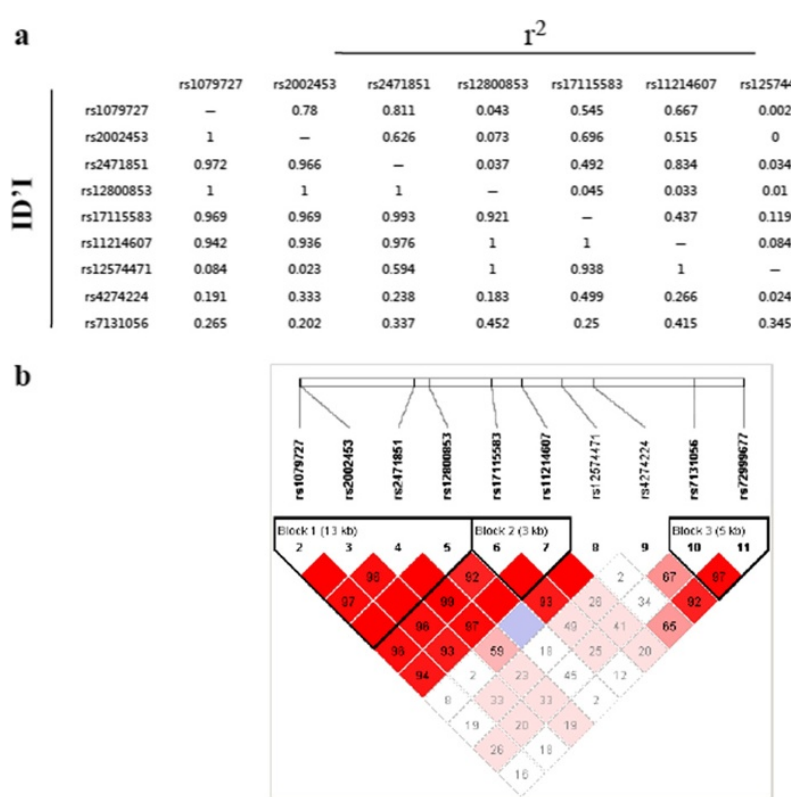

Figure 1 Haplotype analysis of DRD2. (a) Linkage disequilibrium analysis of the 11 SNPs in DRD2 investigated in healthy controls. (b) Three blocks were identified using Haploview software.

memory [38]. Taken together, our data was in accordance with a recent animal study which showed that altered DRD2 expression correlated with selective cognitive impairments in working memory and behavioral flexibility [39]. Working memory represents temporary processing and storage of information, and helps to coordinate different behaviors and functions. It is well documented that dyslexic individuals showed not only impairments with language-specific skills but also working memory defects [40-42]. Hence, it is conceivable that changes in the DRD2 genotype may eventually impair the working memory of dyslexia children in our study.

SLC6A3 contains a 40 base pair variable number of tandem repeats (VNTR) in the 3'-UTR region [43]. The association between the 10-repeat SLC6A3 allele and neurodevelopmental disorders (i.e., ADHD and dyslexia) has 
been reported [25,43-46]. In previous studies, only several SNP markers in the 5 ' region of this gene were identified to be associated with ADHD by SNP genotyping, and none were found in the 3 ' region of the gene, including the 3 ' VNTR and the VNTR of intron $8[47,48]$. In our investigation, we only identified one SNP marker in SLC6A3 showing significant association with dyslexia after adjustment for age and sex, which located in the 3'- untranslated region (3'-UTR). However, the evidence was no longer apparent after Bonferonni adjustment for multiple comparisons. The inconsistent association of SLC6A3 with dyslexia between our study and previous western studies might be explained by linguistic and genetic differences among various populations. English is an alphabetical language while Chinese is logographic. Previous studies found that brain regions associate with dyslexia in western populations and Chinese population might be different. Dyslexia among western populations is associated with dysfunction of left temporoparietal brain regions. Differently, dyslexia among Chinese populations is associated with the left middle frontal gyrus [49,50]. Given the functional differences between these brain regions, the underlying mechanisms of dyslexia among western and Chinese populations might be different as well. But this conclusion requires further validation among larger independent Chinese dyslexia cohort.

\section{Conclusion}

In conclusion, we found significant association between one SNP marker within DRD2 and development dyslexia in a large unrelated Chinese cohort. Our finding supports the involvement of DRD2 polymorphisms in the development of dyslexia. However, further functional analyses are required to explicate its biological roles underlying dyslexia etiology and pathology.

\section{Additional file}

Additional file 1: Table S1. Haplotypes of the three blocks in DRD2 between developmental dyslexia and control subjects. Table S2. Haplotypes of the three blocks in SLC6A3 between developmental dyslexia and control subjects.

\section{Abbreviations}

DRD2: Dopamine D2 receptor; SLC6A3: Solute carrier family 6, member 3 (SLC6A3); FOXP2: Forkhead box P2; DVD: Developmental verbal dyspraxia; CAS: Childhood apraxia of speech; SLI: Speech and language impairment; CNTNAP2: Contactin associated protein-like 2; DYX7: Dyslexia susceptibility locus; MAF: Minor allele frequency; HWE: Hardy-Weinberg equilibrium; LD: Linkage disequilibrium; OR: Odds Ratio.

\section{Competing interests}

The authors declare that they have no competing interests.

\section{Authors' contributions}

YS and LT conceived and designed the experiments; HC, JX, GW and JX performed the experiments; YZ and YG analyzed the data; HC, GW and JX wrote the paper; MY, WS and YJ contributed reagents/materials/analysis tools; all authors read and approved the final manuscript. All authors discussed the results and commented on the manuscript.

\section{Acknowledgements}

This work is funded by the National Key Basic Research Program Grant (2012CB720703). The authors thank all the study subjects, research staff and students who participated in this work.

\section{Author details}

${ }^{1}$ State Key Laboratory of Proteomics, Beijing Proteome Research Center, Beijing Institute of Radiation Medicine, Beijing 102206, China. ${ }^{2}$ National Engineering Research Center for Beijing Biochip Technology, Beijing, China. ${ }^{3}$ CapitalBio Corporation, Beijing, China. ${ }^{4}$ Department of Anatomy, The University of Hong Kong, Hong Kong, China. ${ }^{5}$ State Key Laboratory of Brain and Cognitive Sciences, The University of Hong Kong, Hong Kong, China. ${ }^{6}$ School of Humanities, The University of Hong Kong, Hong Kong, China. ${ }^{7}$ The State Key Laboratory Breeding Base-Shenzhen Key Laboratory of Chemical Biology, The Graduate School at Shenzhen, Tsinghua University, Shenzhen, China. ${ }^{8}$ Neuroimaging Laboratory, Department of Biomedical Engineering, School of Medicine, Shenzhen University, Shenzhen, China. ${ }^{9}$ Guangdong Key Laboratory of Biomedical Information Detection and Ultrasound Imaging, Shenzhen 518060, China. ${ }^{10}$ Medical Systems Biology Research Center, Department of Biomedical Engineering, Tsinghua University School of Medicine, Beijing, China.

Received: 19 May 2014 Accepted: 26 August 2014

Published: 1 September 2014

\section{References}

1. Kang C, Drayna D: Genetics of speech and language disorders. Annu Rev Genomics Hum Genet 2011, 12:145-164.

2. Shaywitz SE: Current concepts - Dyslexia. N Engl J Med 1998, 338(5):307-312

3. Paracchini S, Scerri T, Monaco AP: The genetic lexicon of dyslexia. In Annual Review of Genomics and Human Genetics. Volume 8. Palo Alto: Annual Reviews: Annual Review of Genomics and Human Genetics; 2007:57-79.

4. Saha K, Sambo D, Richardson BD, Lin LM, Butler B, Villarroel L, Khoshbouei $\mathrm{H}$ : Intracellular Methamphetamine Prevents the Dopamine-induced Enhancement of Neuronal Firing. J Biol Chem 2014, 289(32):22246-22257.

5. Foundas AL, Mock JR, Cindass R Jr, Corey DM: Atypical caudate anatomy in children who stutter. Percept Mot Skills 2013, 116(2):528-543.

6. Sasisekaran J: Nonword repetition and nonword reading abilities in adults who do and do not stutter. J Fluency Disord 2013, 38(3):275-289.

7. Lai CS, Fisher SE, Hurst JA, Vargha-Khadem F, Monaco AP: A forkhead-domain gene is mutated in a severe speech and language disorder. Nature 2001, 413(6855):519-523

8. Watkins KE, Dronkers NF, Vargha-Khadem F: Behavioural analysis of an inherited speech and language disorder: comparison with acquired aphasia. Brain 2002, 125(Pt 3):452-464.

9. Tomblin JB, O'Brien M, Shriberg LD, Williams C, Murray J, Patil S, Bjork J, Anderson S, Ballard K: Language features in a mother and daughter of a chromosome 7;13 translocation involving FOXP2. J Speech Lang Hear Res 2009, 52(5):1157-1174.

10. Shriberg LD, Ballard KJ, Tomblin JB, Duffy JR, Odell KH, Williams CA: Speech, prosody, and voice characteristics of a mother and daughter with a 7;13 translocation affecting FOXP2. J Speech Lang Hear Res 2006, 49(3):500-525.

11. MacDermot KD, Bonora E, Sykes N, Coupe AM, Lai CS, Vernes SC, Vargha-Khadem F, McKenzie F, Smith RL, Monaco AP, Fisher SE: Identification of FOXP2 truncation as a novel cause of developmental speech and language deficits. Am J Hum Genet 2005, 76(6):1074-1080.

12. Newbury DF, Monaco AP: Genetic advances in the study of speech and language disorders. Neuron 2010, 68(2):309-320.

13. Turner SJ, Hildebrand MS, Block S, Damiano J, Fahey M, Reilly S, Bahlo M, Scheffer IE, Morgan AT: Small intragenic deletion in FOXP2 associated with childhood apraxia of speech and dysarthria. Am J Med Genet A 2013, 161(9):2321-2326.

14. Zeesman S, Nowaczyk MJ, Teshima I, Roberts W, Cardy JO, Brian J, Senman L, Feuk L, Osborne LR, Scherer SW: Speech and language impairment and oromotor dyspraxia due to deletion of 7q31 that involves FOXP2. Am J Med Genet A 2006, 140(5):509-514. 
15. Condro MC, White SA: Distribution of language-related Cntnap2 protein in neural circuits critical for vocal learning. J Comp Neurol 2014, 522(1):169-185.

16. Rodenas-Cuadrado P, Ho J, Vernes SC: Shining a light on CNTNAP2: complex functions to complex disorders. Eur J Hum Genet 2014, 22(2):171-178

17. Peter B, Raskind WH, Matsushita M, Lisowski M, Vu T, Berninger WW, Wijsman EM, Brkanac Z: Replication of CNTNAP2 association with nonword repetition and support for FOXP2 association with timed reading and motor activities in a dyslexia family sample. J Neurodev Disord 2011, 3(1):39-49.

18. Graham SA, Fisher SE: Decoding the genetics of speech and language. Curr Opin Neurobiol 2013, 23(1):43-51.

19. Pennington BF, Bishop DV: Relations among speech, language, and reading disorders. Annu Rev Psychol 2009, 60:283-306.

20. Gold MS, Blum K, Oscar-Berman M, Braverman ER: Low dopamine function in attention deficit/hyperactivity disorder: should genotyping signify early diagnosis in children? Postgrad Med 2014, 126(1):153-177.

21. Shen $\mathrm{LH}$, Liao MH, Tseng YC: Recent advances in imaging of dopaminergic neurons for evaluation of neuropsychiatric disorders. J Biomed Biotechnol 2012, 2012:259349.

22. Weickert TW, Mattay VS, Das S, Bigelow LB, Apud JA, Egan MF, Weinberger DR, Goldberg TE: Dopaminergic therapy removal differentially effects learning in schizophrenia and Parkinson's disease. Schizophr Res 2013, 149(1-3):162-166.

23. Simonyan $\mathrm{K}$, Horwitz $\mathrm{B}$, Jarvis ED: Dopamine regulation of human speech and bird song: a critical review. Brain Lang 2012, 122(3):142-150.

24. Grigorenko EL: The first candidate gene for dyslexia: Turning the page of a new chapter of research. Proc Natl Acad Sci U S A 2003, 100(20):11190-11192.

25. Lan J, Song M, Pan C, Zhuang G, Wang Y, Ma W, Chu Q, Lai Q, Xu F, Li Y, Liu L, Wang W: Association between dopaminergic genes (SLC6A3 and DRD2) and stuttering among Han Chinese. J Hum Genet 2009, 54(8):457-460.

26. Cornish KM, Savage R, Hocking DR, Hollis CP: Association of the DAT1 genotype with inattentive behavior is mediated by reading ability in a general population sample. Brain Cogn 2011, 77(3):453-458.

27. Hsiung GY, Kaplan BJ, Petryshen TL, Lu S, Field LL: A dyslexia susceptibility locus (DYX7) linked to dopamine D4 receptor (DRD4) region on chromosome 11p15.5. Am J Med Genet B Neuropsychiatr Genet 2004, 125B(1):112-119.

28. Tan LH, Xu M, Chang CQ, Siok WT: China's language input system in the digital age affects children's reading development. Proc Natl Acad Sci US A 2013, 110(3):1119-1123.

29. de Bakker PIW, Yelensky R, Pe'er I, Gabriel SB, Daly MJ, Altshuler D: Efficiency and power in genetic association studies. Nat Genet 2005, 37(11):1217-1223.

30. Barrett JC, Fry B, Maller J, Daly MJ: Haploview: analysis and visualization of LD and haplotype maps. Bioinformatics 2005, 21(2):263-265.

31. Murugan M, Harward S, Scharff C, Mooney R: Diminished FoxP2 levels affect dopaminergic modulation of corticostriatal signaling important to song variability. Neuron 2013, 80(6):1464-1476.

32. Braun AR, Laruelle $M$, Mouradian MM: Interactions between D1 and D2 dopamine receptor family agonists and antagonists: the effects of chronic exposure on behavior and receptor binding in rats and their clinical implications. J Neural Transm 1997, 104(4-5):341-362.

33. Simonyan $K$, Herscovitch $P$, Horwitz B: Speech-induced striatal dopamine release is left lateralized and coupled to functional striatal circuits in healthy humans: a combined PET, fMRI and DTI study. Neuroimage 2013, 70:21-32.

34. Chien YL, Hwu HG, Fann CS, Chang CC, Tsuang MT, Liu CM: DRD2 haplotype associated with negative symptoms and sustained attention deficits in Han Chinese with schizophrenia in Taiwan. J Hum Genet 2013, 58(4):229-232

35. Glatt SJ, Faraone SV, Lasky-Su JA, Kanazawa T, Hwu HG, Tsuang MT: Family-based association testing strongly implicates DRD2 as a risk gene for schizophrenia in Han Chinese from Taiwan. Mol Psychiatry 2009, 14(9):885-893.

36. Nyman ES, Loukola A, Varilo T, Taanila A, Hurtig T, Moilanen I, Loo S, McGough JJ, Jarvelin MR, Smalley SL, Nelson SF, Peltonen L: Sex-specific influence of DRD2 on ADHD-type temperament in a large population-based birth cohort. Psychiatr Genet 2012, 22(4):197-201.

37. Czamara D, Tiesler CM, Kohlbock G, Berdel D, Hoffmann B, Bauer CP, Koletzko S, Schaaf B, Lehmann I, Herbarth O, von Berg A, Müller-Myhsok B, Schulte-Körne G, Heinrich J: Children with ADHD symptoms have a higher risk for reading, spelling and math difficulties in the GINIplus and LISAplus cohort studies. PLoS One 2013, 8(5):e63859.
38. Zhang Y, Bertolino A, Fazio L, Blasi G, Rampino A, Romano R, Lee ML, Xiao T, Papp A, Wang D, Sadee W: Polymorphisms in human dopamine D2 receptor gene affect gene expression, splicing, and neuronal activity during working memory. Proc Natl Acad Sci U S A 2007, 104(51):20552-20557

39. Kellendonk C, Simpson EH, Polan HJ, Malleret G, Vronskaya S, Winiger V, Moore $H$, Kandel ER: Transient and selective overexpression of dopamine D2 receptors in the striatum causes persistent abnormalities in prefrontal cortex functioning. Neuron 2006, 49(4):603-615.

40. Hiscox L, Leonaviciute E, Humby T: The Effects of Automatic Spelling Correction Software on Understanding and Comprehension in Compensated Dyslexia: Improved Recall Following Dictation. Dyslexia 2014, 20(3):108-224.

41. Nergard-Nilssen T, Hulme C: Developmental Dyslexia in Adults: Behavioural Manifestations and Cognitive Correlates. Dyslexia 2014, 20(3):191-207

42. Gabrieli JD: Dyslexia: A New Synergy Between Education and Cognitive Neuroscience. Science 2009, 325(5938):280-283.

43. Cornish KM, Manly T, Savage R, Swanson J, Morisano D, Butler N, Grant C, Cross G, Bentley L, Hollis CP: Association of the dopamine transporter (DAT1) 10/10-repeat genotype with ADHD symptoms and response inhibition in a general population sample. Mol Psychiatry 2005, 10(7):686-698

44. Curran S, Mill J, Tahir E, Kent L, Richards S, Gould A, Huckett L, Sharp J, Batten C, Fernando S, Ozbay F, Yazgan Y, Simonoff E, Thompson M, Taylor E, Asherson P: Association study of a dopamine transporter polymorphism and attention deficit hyperactivity disorder in UK and Turkish samples. Mol Psychiatry 2001, 6(4):425-428.

45. Barr CL, Xu C, Kroft J, Feng Y, Wigg K, Zai G, Tannock R, Schachar R, Malone M, Roberts W, Nöthen MM, Grünhage F, Vandenbergh DJ, Uhl G, Sunohara G, King N, Kennedy JL: Haplotype study of three polymorphisms at the dopamine transporter locus confirm linkage to attention-deficit/hyperactivity disorder. Biol Psychiatry 2001, 49(4):333-339.

46. Chen CK, Chen SL, Mill J, Huang YS, Lin SK, Curran S, Purcell S, Sham P, Asherson P: The dopamine transporter gene is associated with attention deficit hyperactivity disorder in a Taiwanese sample. Mol Psychiatry 2003, 8(4):393-396.

47. Genro JP, Polanczyk GV, Zeni C, Oliveira AS, Roman T, Rohde LA, Hutz MH: A common haplotype at the dopamine transporter gene $5^{\prime}$ region is associated with attention-deficit/hyperactivity disorder. Am J Med Genet B Neuropsychiatr Genet 2008, 147B(8):1568-1575.

48. Doyle C, Brookes K, Simpson J, Park J, Scott S, Coghill DR, Hawi Z, Kirley A, Gill M, Kent L: Replication of an association of a promoter polymorphism of the dopamine transporter gene and Attention Deficit Hyperactivity Disorder. Neurosci Lett 2009, 462(2):179-181

49. Siok WT, Niu Z, Jin Z, Perfetti CA, Tan LH: A structural-functional basis for dyslexia in the cortex of Chinese readers. Proc Natl Acad Sci U S A 2008, 105(14):5561-5566.

50. Siok WT, Perfetti CA, Jin Z, Tan LH: Biological abnormality of impaired reading is constrained by culture. Nature 2004, 431(7004):71-76.

doi:10.1186/1744-9081-10-29

Cite this article as: Chen et al.: Stuttering candidate genes DRD2 but not SLC6A3 is associated with developmental dyslexia in Chinese population. Behavioral and Brain Functions 2014 10:29.

\section{Submit your next manuscript to BioMed Central and take full advantage of:}

- Convenient online submission

- Thorough peer review

- No space constraints or color figure charges

- Immediate publication on acceptance

- Inclusion in PubMed, CAS, Scopus and Google Scholar

- Research which is freely available for redistribution 